\title{
Comparisons of the Millimeter and Submillimeter Bands for Atmospheric Temperature and Water Vapor Soundings for Clear and Cloudy Skies
}

\author{
CATHERINe PRigent \\ Centre National de la Recherche Scientifique, Laboratoire d'Etudes du Rayonnement et de la Matière en Astrophysique, Observatoire \\ de Paris, Paris, France \\ JUAN R. PARDO \\ Departamento de Astrofísica Molecular e Infrarroja, Instituto Estructura de la Materia, Consejo Superior de Investigaciones \\ Científicas, Madrid, Spain \\ WiLLIAM B. Rossow \\ NASA Goddard Institute for Space Studies, New York, New York
}

(Manuscript received 31 May 2005, in final form 29 March 2006)

\begin{abstract}
Geostationary satellites provide revisiting times that are desirable for nowcasting and observations of severe weather. To overcome the problem of spatial resolution from a geostationary orbit, millimeter to submillimeter wave sounders have been suggested. This study compares the capabilities of various oxygen and water vapor millimeter and submillimeter bands for temperature and water vapor atmospheric profiling at nadir in cloudy situations. It shows the impact of different cloud types on the received signal for the different frequency bands. High frequencies are very sensitive to the cloud ice phase, with potential applications to cirrus characterization.
\end{abstract}

\section{Introduction}

Operational meteorological satellites in polar orbits make microwave measurements in the $\mathrm{O}_{2}$ band around $60 \mathrm{GHz}$ and in the $\mathrm{H}_{2} \mathrm{O}$ line at $183.31 \mathrm{GHz}$ for atmospheric temperature and water vapor sounding, respectively. These measurements complement infrared (IR) observations that are generally limited to cloud-free areas.

For nowcasting and observations of severe weather, higher satellite orbits are suggested to provide the required revisit times. The problem is that adequate spatial resolution on the ground cannot be obtained at lower microwave frequencies with a feasible antenna size because geosynchronous orbit altitudes are about

Corresponding author address: Catherine Prigent, Centre National de la Recherche Scientifique, Laboratoire d'Etudes du Rayonnement et de la Matière en Astrophysique, Observatoire de Paris, 61, avenue de l'Observatoire, Paris, France.

E-mail: catherine.prigent@obspm.fr
45 times larger than polar orbit altitudes. One solution is to use higher frequencies.

So far, in the framework of operational meteorological satellites, passive microwave observations have been limited to frequencies of up to $190 \mathrm{GHz}$. Technological advances now make it possible to operate reliable radiometers at higher microwave frequencies. A number of research missions employing submillimeter wavelengths exist for either upper-atmospheric tracegas spectroscopy or for astronomy. For example, the second Microwave Limb Sounder (MLS) was launched in July 2004 on Earth Observing System (EOS) Aura with frequencies of up to $2.5 \mathrm{THz}$ (Waters et al. 1999). Odin, a small Swedish satellite dedicated to the study of both astronomical objects and the earth's upper atmosphere, was launched in February 2001 and also employs frequencies of up to $600 \mathrm{GHz}$.

Millimeter and submillimeter instruments for geostationary satellites are now planned for meteorological applications, following pioneering studies from Chédin et al. (1986) and Gasiewski (1992), for example. The National Aeronautics and Space Administration 
(NASA) National Oceanic and Atmospheric Administration (NOAA) Geosynchronous Microwave Sounder Working Group developed the project of a submillimeter-wave geosynchronous microwave (GEM) sounder and imager equipped with a 2-m scanning antenna, with channels in the oxygen bands $(54,118$, and $425 \mathrm{GHz})$ and in the water vapor lines (183 and $340 / 380 \mathrm{GHz}$ ) (Staelin et al. 1998). The Geostationary Observatory for Microwave Atmospheric Sounding (GOMAS) project was also proposed recently to the European Space Agency by B. Bizzari et al. (2005, personal communication) as a Next Earth Explorer core mission, with channels similar to those of GEM. Millimeter and submillimeter observations are also suggested from polar satellites to help characterize the ice clouds (e.g., Evans et al. 1999; Goutoule et al. 2003; S. Buehler et al. 2005, personal communication).

This study evaluates the capabilities of higher frequencies in the millimeter and submillimeter ranges for temperature and water vapor profiling, even for the case where clouds are present. Klein and Gasiewski (2000) already conducted a thorough analysis of clearsky nadir sensitivities of the millimeter and submillimeter ranges. Our study extends the analysis to cloudy sky, estimating potential cloud contamination in the various absorption bands using cloud climatological information. The microwave radiative transfer simulations are performed with the Atmospheric Transmission at Microwave (ATM) model (see references in section 2) that can handle both clear and cloudy situations. The continuum and $\mathrm{O}_{2}$ and $\mathrm{H}_{2} \mathrm{O}$ lines in this model have been validated with observations up to $1.5 \mathrm{THz}$. The model and the related validation observations are briefly described in section 2 . Absorption bands up to $1000 \mathrm{GHz}$ are analyzed in section 3 for their capabilities for 1) temperature and 2) water vapor profiling. In section 4, cloud statistics derived from the International Satellite Cloud Climatology Project (ISCCP) (Rossow and Schiffer 1999) help to estimate the percentage of time that each frequency band will actually be affected by clouds. Section 5 summarizes this analysis.

\section{The ATM radiative transfer model}

The ATM model has been developed during the past 20 years for applications in both ground-based astronomy and Earth remote sensing. The model is applicable from 0 to $2 \mathrm{THz}$. It can simulate close-to-nadir and limb observations from satellite platforms (Pardo et al. 2001a,b, 2002, 2004; Prigent et al. 2001; Garand et al. 2001; Ridal et al. 2002). The model is composed of the following four basic parts: the absorption by atmospheric gases, the scattering calculations for spherical and nonspherical hydrometeors, the surface emissivity estimated above ocean and land, and the radiative transfer through an atmosphere with plane-parallel layers.

Line-by-line calculations of the resonant part of the absorption are performed using a line atlas generated from the latest available spectroscopic constants for all relevant atmospheric species (Pardo et al. 2001a, and references therein). The excess of absorption in the "longwave" range that cannot be explained by the resonant spectrum is modeled by introducing continuumlike terms. Using the spectroscopy described in Pardo et al. (2001a) and ground-based Fourier transform spectroscopy measurements, the continuum terms that have to be added to the line spectrum have been derived (Pardo et al. 2001b, 2004). The results are summarized in Pardo et al. (2005).

The model treats the scattering of radiation through layers of hydrometeors as described in Prigent et al. (2001, 2005a) and Wiedner et al. (2004). The absorption of atmospheric gases is first calculated in all layers with the clear-sky routines, and then the far-field scattering by single spherical or nonspherical particles is computed with the T-matrix algorithm developed by Mishchenko (1991, 1993, 2000). In this work, spherical particles are considered, but the case of partially oriented nonspherical particles (provided that the orientation is random at least in azimuth) can be treated. Different particle size distributions can be selected (e.g., lognormal or modified gamma). The refractive index is taken from Manabe et al. (1987) for liquid water droplets and from Warren (1984) for ice particles.

The model also includes a full treatment of the effect of the surface (Lambertian or Fresnel-like surfaces), as well as a model to treat the wind-roughened ocean surface (Guillou et al. 1996). A land surface emissivity atlas derived from Special Sensor Microwave Imager (SSM/I) and Advanced Microwave Sounding Unit (AMSU) observations is attached to the model (Prigent et al 1997, 2005b), along with an angular and frequency dependence parameterization.

The final radiative transfer calculation is performed using the "doubling and adding" method as described in Evans and Stephens (1995).

\section{Temperature and water vapor profiling in clear sky}

Figure 1 represents the clear-sky atmospheric opacity at nadir for frequencies of up to $1 \mathrm{THz}$, as simulated with the ATM model for a mean standard atmosphere (integrated water vapor content of $14.3 \mathrm{~kg} \mathrm{~m}^{-2}$ and surface temperature of $28.7 \mathrm{~K}$ ). In the millimeter and 


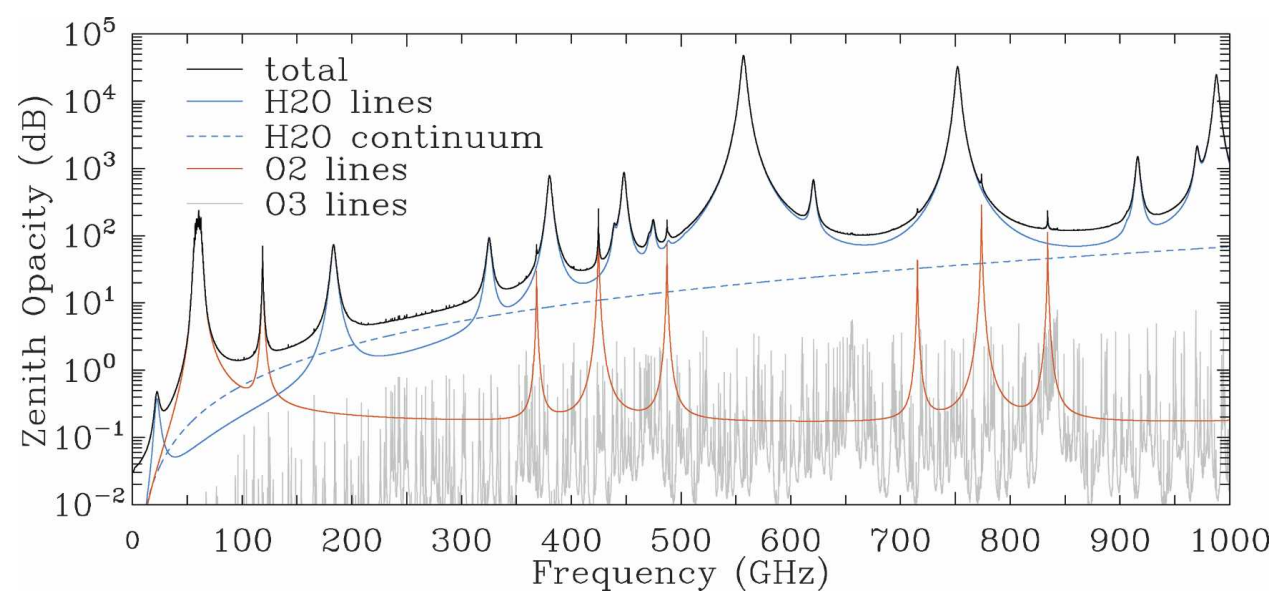

FIG. 1. Clear-sky atmospheric opacity at nadir for up to $1 \mathrm{THz}$ for a mean standard atmosphere.

submillimeter ranges, there are a large number of $\mathrm{H}_{2} \mathrm{O}$ lines, most of them stronger than the lines below 200 $\mathrm{GHz}$. In this high-frequency domain $\mathrm{O}_{2}$ has a number of isolated strong lines as well, with absorption characteristics similar to that of the $118-\mathrm{GHz}$ line. However, with increasing frequency, the increasing water vapor continuum strength, combined with the strong wings of the $\mathrm{H}_{2} \mathrm{O}$ absorption lines, increases the opacity between the lines, thus limiting the possibility of lowertropospheric soundings at these higher frequencies. The sensitivity of each frequency to the atmospheric parameters is assessed in terms of the changes of the measured brightness temperature at the top of the atmosphere for a small perturbation of the given atmospheric parameter. The weighting functions $\mathrm{Wp}$ (also called the incremental weighting functions, see Klein and Gasiewski 2000) are defined as follows:

$$
d \operatorname{Tb}(f, \theta)=\int_{0}^{\infty} d x(z) \mathrm{Wp}(z, f, \theta) d z,
$$

where $d \mathrm{~Tb}$ is the brightness temperature change at frequency $f$ for observation angle $\theta$ with respect to nadir resulting from a variation of $d x(z)$ in the atmospheric parameter $x$ at altitude $z$.

\section{a. Temperature profiling}

In addition to the spin-rotation band around $60 \mathrm{GHz}$, $\mathrm{O}_{2}$ absorption exhibits seven isolated lines at 118.750345 , 368.498 440, 424.763 123, $487.249381,715.393320$, 773.839669 , and $834.145728 \mathrm{GHz}$. Figure 2 zooms on $20 \mathrm{GHz}$ around the five lowest frequencies of the above list showing their total nadir opacity (lower panel) for three different atmospheres, along with the $\mathrm{O}_{2}$ and the $\mathrm{O}_{3}$ opacity. Even for a dry atmosphere (e.g., subarctic in Fig. 2), the $\mathrm{H}_{2} \mathrm{O}$ opacity is large for frequencies above $200 \mathrm{GHz}$, thus limiting the possibility of a loweratmospheric sounding in this frequency range. The three higher-frequency bands of $\mathrm{O}_{2}$ above $700 \mathrm{GHz}$ are not presented in this figure because they are even more affected by $\mathrm{H}_{2} \mathrm{O}$ absorption. In contrast, the opacity in the band around $60 \mathrm{GHz}$ is not significantly affected by water vapor changes as the environment changes from subarctic to tropical.

Figure 2 (upper panel) shows the corresponding monochromatic weighting functions for selected frequencies around the line centers, calculated for nadir soundings over land for a mean standard atmosphere with the land surface emissivity assumed to unity as an illustration. Farther from the line center, where the opacity variations with frequency are limited, the weighting functions do not change significantly with frequency either and no further calculations are performed. The frequency band around $368.4984 \mathrm{GHz}$ is clearly not suitable for temperature profiling resulting from low sensitivities to changes in temperature in the higher atmosphere (very broad weighting function with maximum around 0.5 ). Around $400 \mathrm{hPa}$, the weighting functions are narrower with a large maximum but are related to the water vapor absorption (see the corresponding opacities) and as such they will be very sensitive to water vapor changes. Down to $300 \mathrm{hPa}$, the weighting functions are similar for the 60-, 118-, 424-, and $487-\mathrm{GHz}$ bands. The sensitivity of the $424-\mathrm{GHz}$ band to changes in temperature is slightly larger, with a maximum of the weighting function of $\sim 0.12 \mathrm{~K} \mathrm{~K}^{-1}$ $\mathrm{km}^{-1}$ as compared with $\sim 0.1 \mathrm{~K} \mathrm{~K}^{-1} \mathrm{~km}^{-1}$ for the other bands, resulting from the larger line strength relative to the other isolated lines. In the lower atmosphere, the $\mathrm{H}_{2} \mathrm{O}$-related opacity at frequencies above $200 \mathrm{GHz}$ prevents sounding of the lower atmosphere. At these frequencies, the large maxima of the weighting func- 

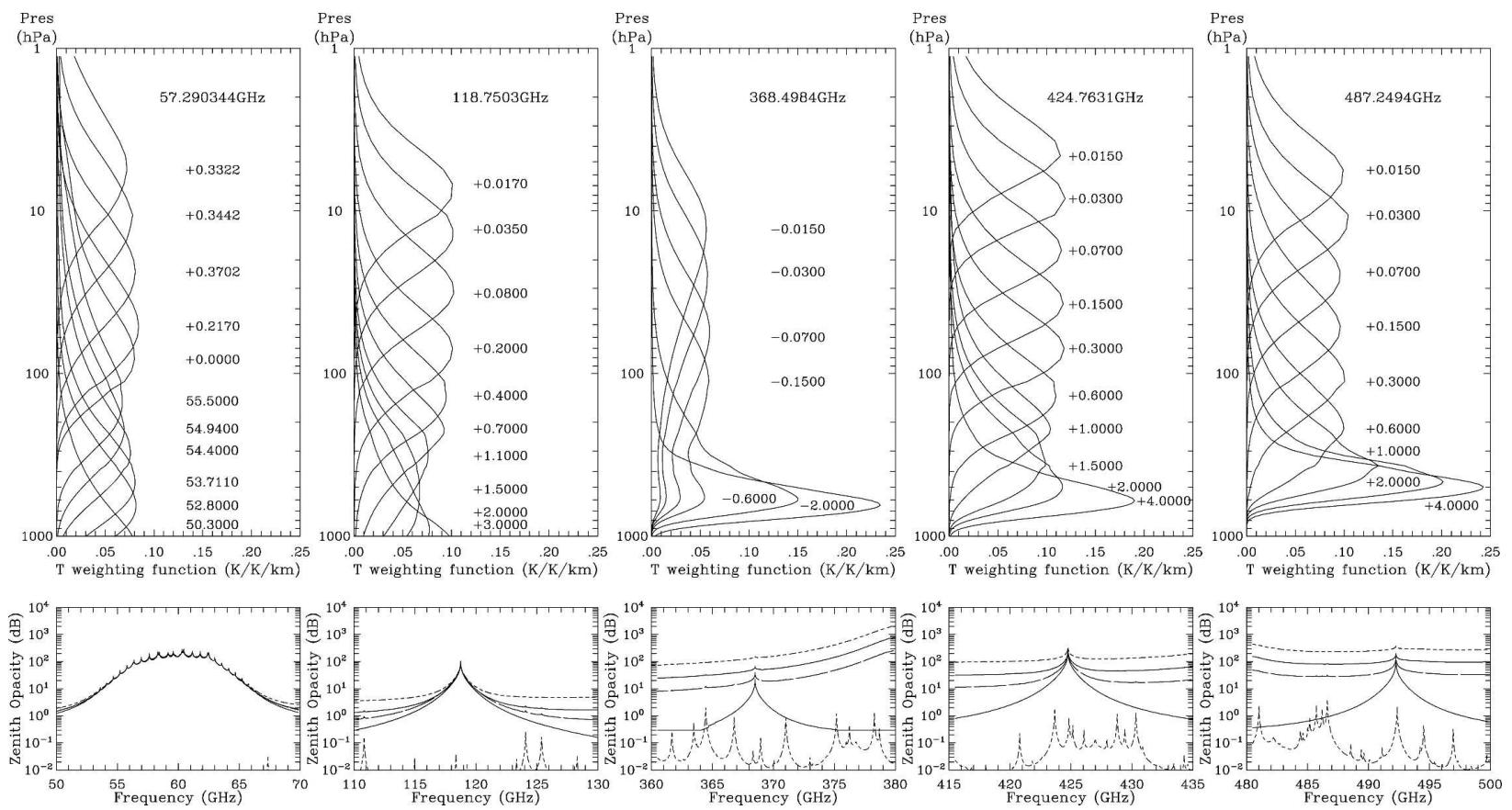

FIG. 2. For each of the five lower $\mathrm{O}_{2}$ bands, (bottom) the total zenith opacity for three different atmospheres (subarctic, long dashed lines; mean standard, solid line; tropical, short dashed lines), along with the $\mathrm{O}_{2}$ (solid gray line) and the minor components opacities (dashed gray lines), and (top) the corresponding monochromatic weighting functions for selected frequencies around the line centers, calculated for nadir sounding over land (surface emissivity of unity) for a mean standard atmosphere.

tions in the lower atmosphere are only a consequence of the sensitivity of the water vapor absorption to temperature. Comparisons between the $60-$ and $118-\mathrm{GHz}$ weighting functions show similar performances, although the weighting functions at $118 \mathrm{GHz}$ are slightly broader than those at $60 \mathrm{GHz}$, potentially resulting in a slightly degraded vertical resolution of the sounding, especially in the lower atmosphere. The $118-\mathrm{GHz}$ channels have already been advocated by Gasiewski and Johnson (1993).

In the following, only the 60-, 118-, and $424-\mathrm{GHz}$ bands will be further analyzed, with the other bands being inappropriate for temperature sounding of the lower atmosphere.

\section{b. Water vapor profiling}

The atmospheric absorption spectrum below $1 \mathrm{THz}$ shows a large number of rotational water vapor lines, among which is the lowest rotational transition of orthowater at $556.9361 \mathrm{GHz}$. In this study, only the lines at 183.3100, 325.1528, 380.1976, 448.0013, and $556.9361 \mathrm{GHz}$ are considered; the other lines are too weak for adequate profiling and/or are located at higher frequencies where the water vapor continuum plus the strong line wings make the opacity too large between lines. In Fig. 3, similar to Fig. 2 for $\mathrm{O}_{2}$, the total nadir opacity at the selected $\mathrm{H}_{2} \mathrm{O}$ central frequencies is shown (lower panel) for three different atmospheres, along with the $\mathrm{O}_{2}$ and the $\mathrm{O}_{3}$ opacities (note that the vertical scale is not the same for the highest frequency). The water vapor weighting functions are presented in the upper panel for the corresponding bands. The 183and $325-\mathrm{GHz}$ bands have similar opacities, with a larger opacity in the wings of the $325-\mathrm{GHz}$ line. However, by properly selecting the frequency, similar weighting functions can be obtained above $800 \mathrm{hPa}$. Close to the surface, both frequencies experience problems with limited sensitivity to the lower atmosphere. The 380and 448-GHz bands exhibit higher opacities close to the line centers and, as a consequence, sounding at higher altitudes is possible (up to $\sim 250 \mathrm{hPa}$ ). The strong 556GHz line does not provide additional sounding capacity for an atmosphere that is dry at high altitudes.

\section{Cloud impact on the selected $\mathrm{O}_{2}$ and $\mathrm{H}_{2} \mathrm{O}$ bands}

\section{a. Cloud climatological data}

Since 1983, ISCCP collects, calibrates, navigates, and analyzes the infrared $(\sim 11 \mu \mathrm{m})$ and visible radiance $(\sim 0.65 \mu \mathrm{m})$ from the radiometers on board the international weather satellites, both on polar and geosta- 

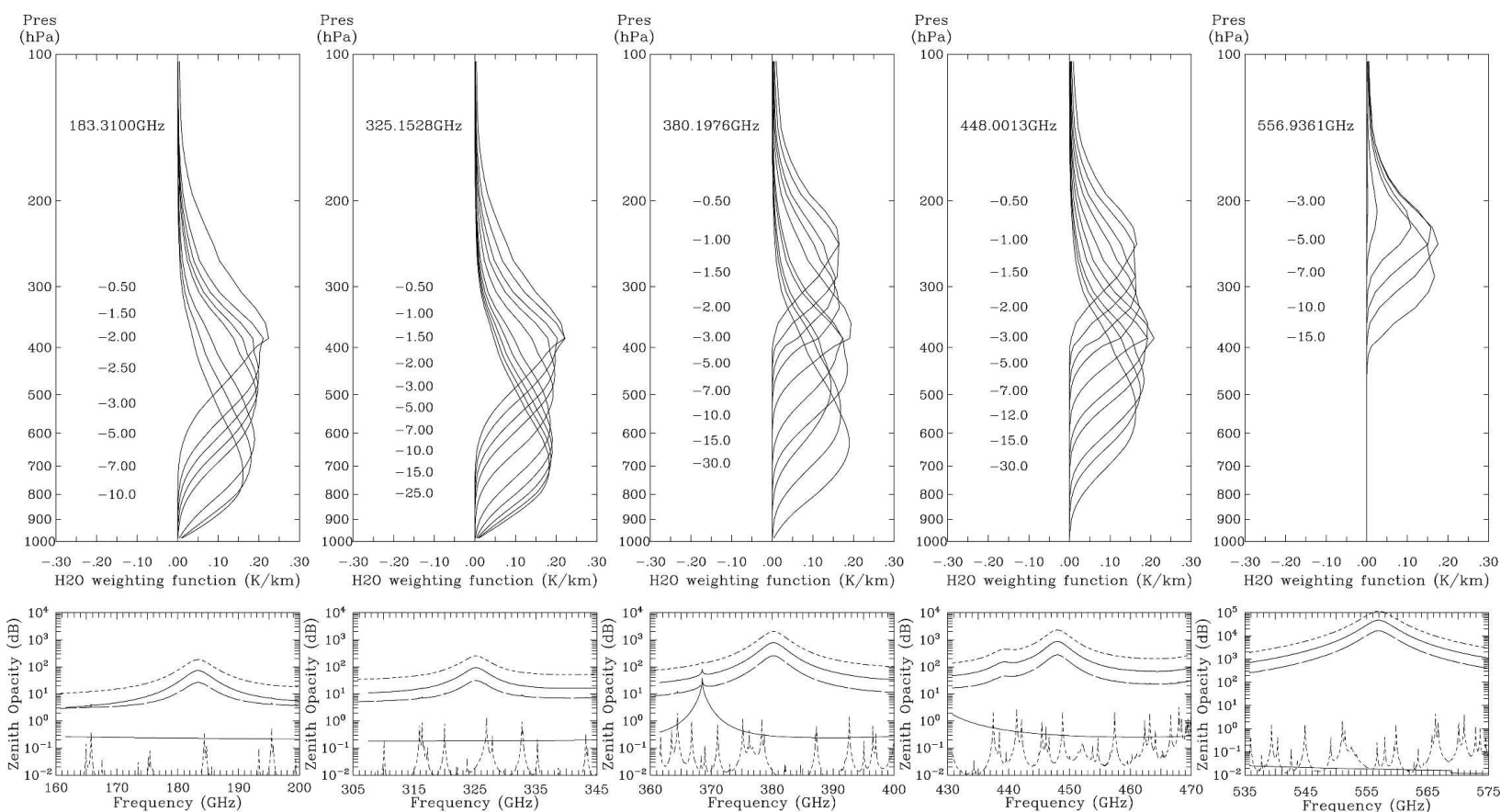

FIG. 3. Same as in Fig. 2, but for $\mathrm{H}_{2} \mathrm{O}$ lines.

tionary orbits. The objective is to characterize the main cloud properties and their temporal and spatial variations over the globe. More than 20 years of data are now available (July 1983-December 2004). Cloud fraction is determined every $3 \mathrm{~h}$ for areas of $280 \mathrm{~km} \times 280$ $\mathrm{km}$ (equivalent to $2.5^{\circ} \times 2.5^{\circ}$ at the equator), estimated from the $5 \mathrm{~km} \times 5 \mathrm{~km}$ original dataset that is sampled every $30 \mathrm{~km}$. The cloud detection process is described and validated in Rossow and Schiffer (1991) and Rossow and Garder (1993a,b), and the cloud amount in Rossow and Garder (1993b). Cloud-top temperature and pressure are derived from the infrared observations. Cloud optical thicknesses are retrieved from visible measurements using two different cloud microphysical models - one for liquid particles and one for ice particles, when the cloud top temperature is above or below $260 \mathrm{~K}$, respectively. Recent ISCCP results are discussed in Rossow and Schiffer (1999) (see also Rossow and Duenas 2004). Nine cloud types are defined, based on their optical thickness and cloud-top pressure; they are named from the classic morphological cloud types. Table 1 gives the ISCCP average mean global cloud amount for 1986-93 for each cloud type, separating them by the phase of the cloud particles (liquid or ice) and by their occurrence over land and ocean (with the first number in parentheses representing ocean, the second one land).

The global cloud amount is $\sim 67 \%$. Clouds are almost equally divided into liquid and ice clouds $(33.5 \%$ and
$32.8 \%$, respectively). Thin clouds that are likely to be transparent at millimeter wavelengths amount to $35 \%$. Very thick clouds only represent $\sim 7 \%$ of the total. Large differences are observed between ocean and land cloud amounts $(\sim 10 \%$ difference). Significant variations with latitude are also observed (Fig. 4), with midlatitude cloud amount exceeding that in the Tropics, except for high clouds.

\section{b. Cloud impact on each frequency band}

The cloud impact at each frequency will depend not only upon the cloud optical thickness but also on its phase and altitude. Depending on the wavelength and the cloud characteristics, the radiation will be affected by emission, absorption, and scattering. In the millimeter and submillimeter ranges, emission and absorption are the dominant mechanisms involved in the interaction between the radiation and nonprecipitating liquid droplets; consequently, the effects are not sensitive to particle size directly. In contrast, ice particles do not absorb much so scattering effects prevail, with intensities increasing with increasing frequencies.

For a given frequency, clouds that are at altitudes above or close to the maximum of the weighting function will obviously affect this channel the most. To quantify the impact of clouds on the measurements, radiative transfer simulations are performed that include clouds in the atmospheric column. The objective of this study is to derive representative statistical esti- 
TABLE 1. Global cloud statistics derived from ISCCP: average mean global cloud amount for 1986-93 for each cloud type, separating them by the phase of the cloud particles (liquid or ice) and by their occurrence over land and ocean (the first number in parentheses represents ocean, and the second number represents land). Here, high clouds correspond to cloud-top pressure of 50-440 hPa, middle clouds to cloud-top pressure of 440-680 hPa, and low clouds to cloud-top pressures of 680-1000 hPa. Thin clouds correspond to cloud visible optical thickness of 0-3.6, medium clouds correspond to cloud visible optical thickness of 3.6-23, and thick clouds correspond to cloud visible optical thickness of 23-379.

\begin{tabular}{|c|c|c|c|}
\hline High clouds: & Cirrus: & Cirrostratus: & Deep convection: \\
\hline Total $21.6(20.7-23.6)$ & Total $13.2(12.0-15.8)$ & Total $5.8(6.0-5.2)$ & Total $2.6(2.7-2.5)$ \\
\hline Liquid $0.0(0.0-0.0)$ & Liquid $0.0(0.0-0.0)$ & Liquid $0.0(0.0-0.0)$ & Liquid $0.0(0.0-0.0)$ \\
\hline Ice $21.6(20.7-23.6)$ & Ice $13.2(12.0-15.8)$ & Ice $5.8(6.0-5.3)$ & Ice $2.6(2.7-2.5)$ \\
\hline Middle clouds: & Altocumulus: & Altostratus: & Nimbostratus: \\
\hline Total $19.2(19.3-19.2)$ & Total $9.3(9.8-8.4)$ & Total $7.8(7.7-8.0)$ & Total $2.1(1.8-2.8)$ \\
\hline Liquid $9.3(9.6-8.4)$ & Liquid $4.2(4.8-2.9)$ & Liquid $4.0(3.9-4.0)$ & Liquid $1.1(0.9-1.5)$ \\
\hline Ice $9.9(9.7-10.8)$ & Ice $5.1(5.0-5.5)$ & Ice $3.8(3.8-4.0)$ & Ice $1.0(0.9-1.3)$ \\
\hline Low clouds: & Cumulus: & Stratocumulus: & Stratus: \\
\hline Total $25.5(30.6-17.4)$ & Total $12.5(14.5-8.0)$ & Total $12.121 .1-6.6)$ & Total $1.9(2.0-1.8)$ \\
\hline Liquid 24.2 (28.2-15.0) & Liquid 11.3 (13.2-6.8) & Liquid 11.2 (13.2-6.7) & Liquid 1.7 (1.8-1.5) \\
\hline Ice $2.3(2.4-2.4)$ & Ice $1.2(1.3-1.2)$ & Ice $0.9(0.9-0.9)$ & Ice $0.2(0.2-0.3)$ \\
\hline All clouds: & Thin clouds: & Medium clouds: & Thick clouds: \\
\hline Total $67.3(70.6-60.2)$ & Total $35.0(36.3-32.2)$ & Total 25.7 (27.8-20.9) & Total $6.6(6.5-7.1)$ \\
\hline Liquid 33.5 (37.8-23.4) & Liquid 15.5 (18.0-9.7) & Liquid $15.2(17.1-10.7)$ & Liquid 2.8 (2.7-3.0) \\
\hline Ice $32.8(32.8-36.8)$ & Ice $19.5(18.3-22.2)$ & Ice $10.5(10.7-10.2)$ & Ice $3.8(3.8-4.1)$ \\
\hline
\end{tabular}

mates of different cloud-type perturbations on the radiometric signal, not to describe the full range of variability that can be encountered in cloudy situations. The ISCCP cloud climatological data are used. Outputs from cloud mesoscale models would give more detailed descriptions of specific cloud situations, but would not be representative of the average cloud statistics over the globe. Differences in brightness temperatures are calculated between cloudy and clear skies for the following six types of clouds, as specified by ISCCP: low liquid cloud, middle liquid cloud, and high ice cloud, for both thin and medium cases. Cloud-top pressure and cloud optical thickness are selected to match the ISCCP cloud classification. Cloud optical thickness is converted into liquid (ice) water path, assuming a cloud particle effective radius of $10-\mu \mathrm{m}(30 \mu \mathrm{m})$ radius, a normalized Mie extinction coefficient of 2.119 (2.0), and a density of $1 \mathrm{~g} \mathrm{~cm}^{-3}\left(0.525 \mathrm{~g} \mathrm{~cm}^{-3}\right)$. The liquid (ice) water path $\left(\mathrm{kg} \mathrm{m}^{-2}\right)$ is thus $6.292 \times 10^{-3}(10.500$ $\times 10^{-3}$ ) times the optical thickness (Rossow et al. 1996). The magnitude of the water path estimated from the ISCCP results depends critically on the assumed particle size; on average, the ISCCP value of $10 \mu \mathrm{m}$ is within 1-2 $\mu \mathrm{m}$ of the values determined from satellite surveys (Han et al 1994, 1995), so the water path values are within $10 \%-20 \%$. This effective radius for liquid droplets has been evaluated during measurement campaigns [the First ISCCP Regional Experiment (FIRE)] and compared with other estimates (Han et al. 1995). It is also commonly adopted in mesoscale cloud models. In the case of ice clouds, it is not the ice water path that matters much but the scattering optical depth with weaker size dependence for the millimeter and submillimeter wavelengths than for the wavelength range used in ISCCP. The ISCCP ice particle size of $30 \mu \mathrm{m}$ is likely to be an underestimate because available satellite measurements apply to the tops of these clouds and the larger particles occur nearer the cloud base. From a global analysis using the Television and Infrared Observation Satellite (TIROS)-N Operational Vertical Sounder, Stubenrauch et al. (1999) found a 35-45- $\mu \mathrm{m}$ average effective radius for thin cirrus. The sensitivity of the results to the assumed particle size is shown below.

In our simulations, monodispersed particles of 10 and $30-\mu \mathrm{m}$ radius are used for liquid and ice particles, respectively, for consistency with the ISCCP climatological data. Simulations have also been performed using larger ice particles (radius of $60 \mu \mathrm{m}$ ) to illustrate the effect of particle sizes on the scattering. However, this particle size represents an upper limit for medium ice clouds and is unlikely for thin cirrus.

Calculations are performed for the $\mathrm{O}_{2}$ and $\mathrm{H}_{2} \mathrm{O}$ bands over ocean and land for a mean standard atmosphere. The ocean has a low emissivity, whereas the land surface emissivities are often close to unity. The cloud impacts have been calculated for emissivity 0.5 and 1 that are representative of the extreme emissivity 

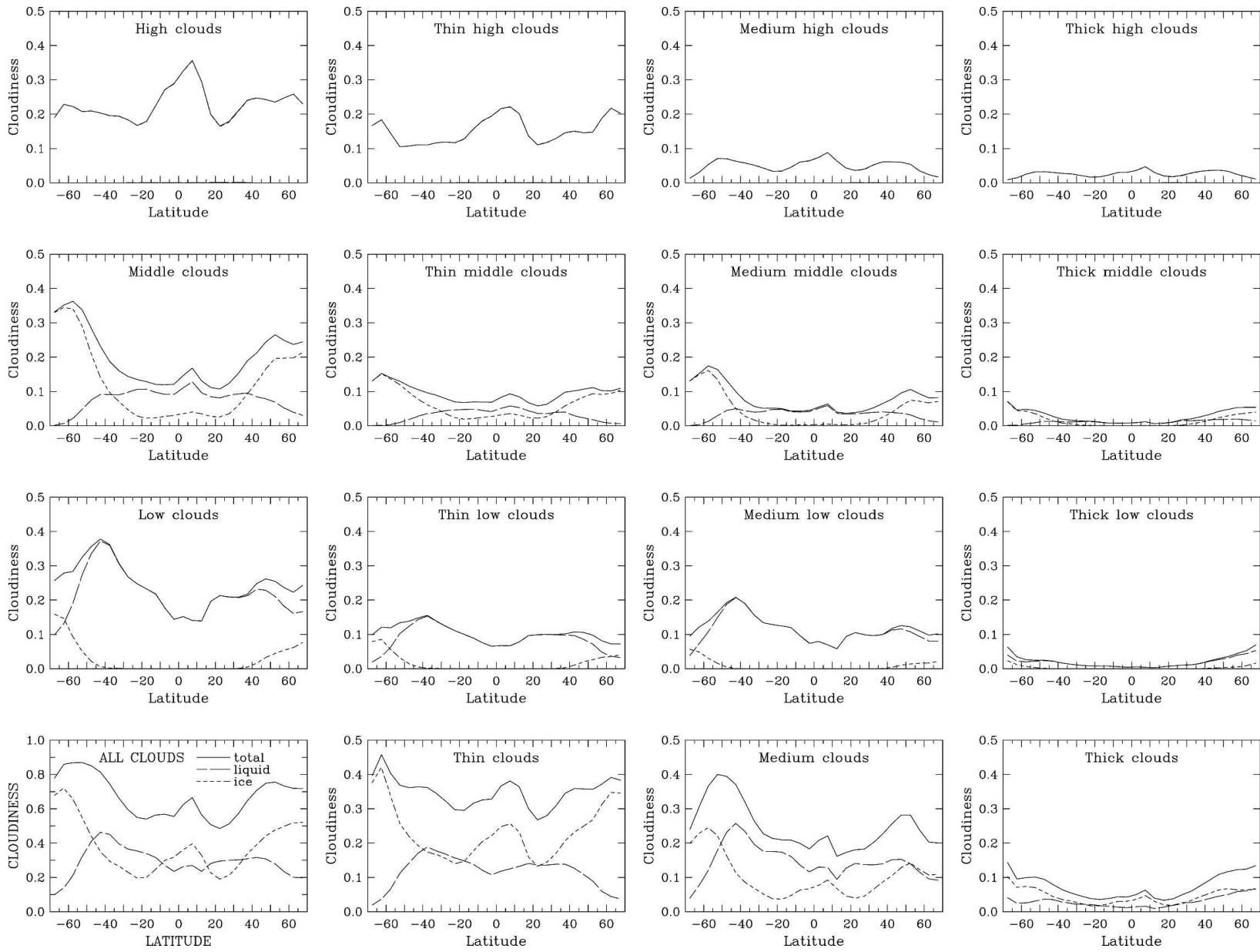

FIG. 4. Latitudinal cloud statistics derived from ISCCP.

values. For intermediate values, the results are expected to be within the range obtained with these two values. The cloud statistics that are used in the following discussion are based on global annual mean data. For a satellite that would focus on a specific region, the results can be quantitatively different (see Fig. 4).

\section{1) $\mathrm{O}_{2}$ LINES}

Figure 5 presents the effect of the clouds (Tb cloudy sky minus $\mathrm{Tb}$ clear sky) in the $\mathrm{O}_{2}$ bands. Thin clouds (visible optical thickness below 3.6) that represent 35\% of total cases (i.e., $~ 50 \%$ of the cloudy cases) affect the $60-\mathrm{GHz}$ bands by less than $0.5 \mathrm{~K}$ over land or over ocean. In contrast, thin clouds (medium or high) do affect the $425-\mathrm{GHz}$ band. Low clouds do not affect this band just because the total gas opacity is so large that it cannot sound the lower atmosphere. Medium ice clouds (visible optical thickness between 3.6 and 23), 10\% of the total cases, impact the $60-$ and $118-\mathrm{GHz}$ useful bands by less than $1 \mathrm{~K}$. The medium liquid clouds $(\sim 15 \%)$ and the thick clouds $(\sim 7 \%)$ seriously affect those bands. In any case, the channels that sound levels above $\sim 200 \mathrm{hPa}$ (i.e., above most clouds) will rarely be affected. However, for medium liquid clouds, the 118$\mathrm{GHz}$ channels will be more affected than the $60-\mathrm{GHz}$ channels, with measurable changes in brightness temperatures starting at $\sim 0.7 \mathrm{GHz}$ from the $118-\mathrm{GHz}$ line center (i.e., channels with weighting function peaks as low as $200 \mathrm{hPa}$ ), whereas the $60-\mathrm{GHz}$ weighting functions will not be significantly affected below $\sim 400 \mathrm{hPa}$, down to the surface.

It is worth mentioning that with increasing frequencies (and usually increasing atmospheric absorption), the surface effects are considerably diminished. This can be clearly observed in Fig. 5 where the effect of the surface emissivity on the sensitivity to clouds (regardless of the cloud types) decreases with increasing frequencies: at low frequencies the sensitivity to clouds depends upon the surface type, whereas at high frequencies it is very similar regardless of the surface type.

Figure 6 shows the effect of ice particle sizes on the scattering, with a comparison of the ice cloud effect for 

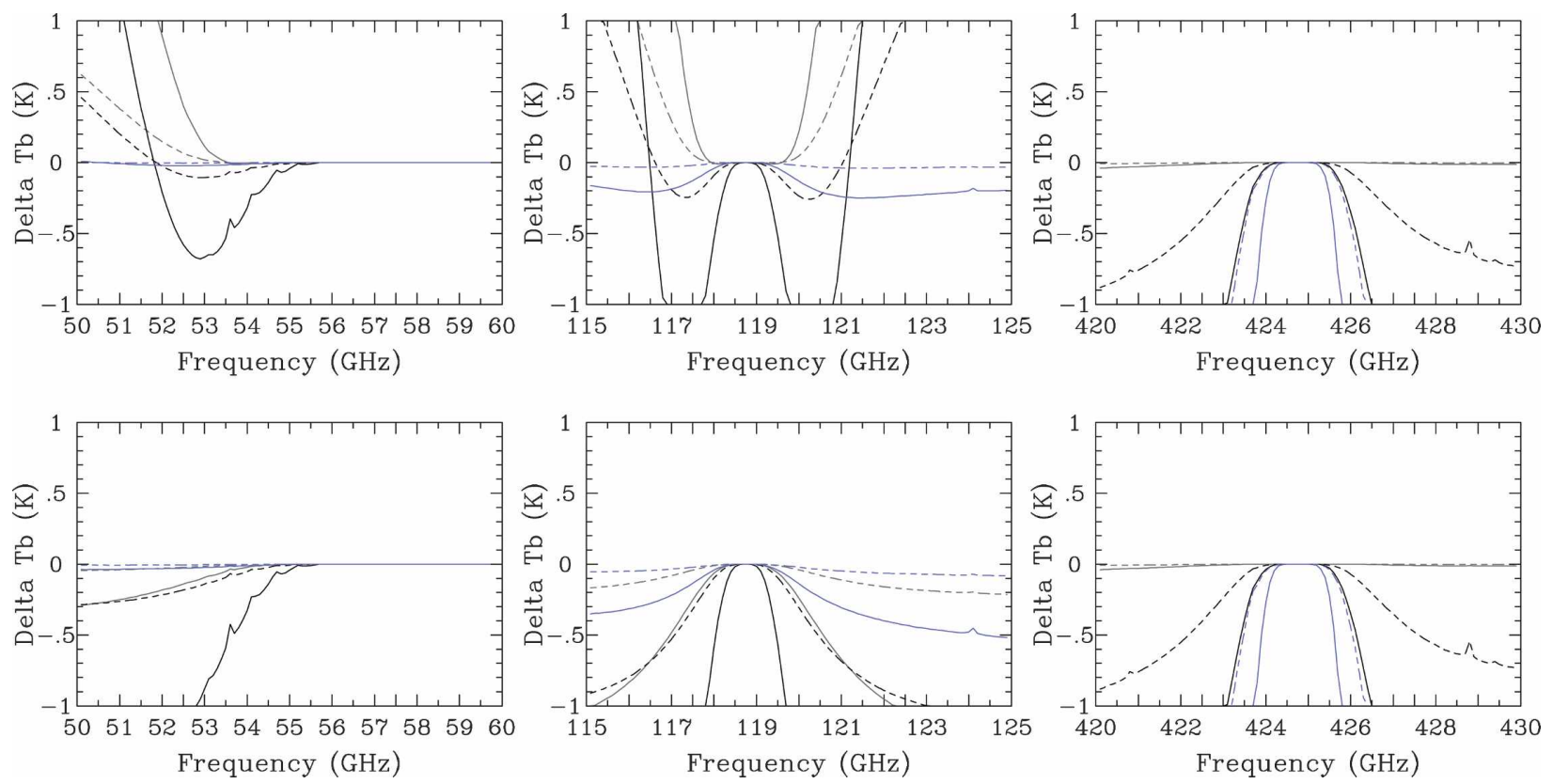

FIG. 5. Sensitivity of $\mathrm{O}_{2}$ lines to clouds (DeltaTb $=\mathrm{Tb}$ cloudy sky minus Tb clear sky) for a standard mean atmosphere (top) over ocean (emissivity $=0.5$ ) and (bottom) over land (emissivity $=1.0)$ : low liquid clouds $(900-800 \mathrm{hPa}$, gray lines), middle liquid clouds (550-480 hPa, black lines), high ice clouds (360-300 hPa, blue lines), thin clouds (visible optical thickness $=3.6$, i.e., LWP $=0.022 \mathrm{~kg}$ $\mathrm{m}^{-2}$ or IWP $=0.037 \mathrm{~kg} \mathrm{~m}^{-2}$, dashed lines), and medium clouds (visible optical thickness $=23$, i.e., $\mathrm{LWP}=0.144 \mathrm{~kg} \mathrm{~m} \mathrm{~m}^{-2}$ or IWP $=$ $0.241 \mathrm{~kg} \mathrm{~m}^{-2}$, solid lines).

two particle radii $(30$ and $60 \mu \mathrm{m})$. Around $60 \mathrm{GHz}$, the cloud effect is very limited, regardless of the particle size, but at $118 \mathrm{GHz}$, with increasing particle size, the scattering effect increases, especially for medium clouds. At $424 \mathrm{GHz}$, the scattering is already important for small particle sizes and a saturation effect occurs.

The sensitivity of high frequencies to ice cloud properties, including ice content and particle size, makes it possible to detect and characterize ice clouds with such channels. This has been discussed in various studies (e.g., Evans et al. 2002; Miao et al. 2003; Davis et al. 2005).

\section{2) $\mathrm{H}_{2} \mathrm{O}$ LINES}

The effect of the clouds (Tb cloudy sky minus $\mathrm{Tb}$ clear sky) in the $\mathrm{H}_{2} \mathrm{O}$ bands is shown in Fig. 7. The impact of clouds is very similar over land and ocean, for all channels, because of the large gas opacities of these channels and, consequently, their relative insensitivity to the lower atmosphere. Clouds affect a channel when they are located at an altitude where the channel weighting function is not negligible (see the weighting function Fig. 3). Low clouds do not affect these three frequency bands much because of the small contribution from the lower atmosphere to the measured radiance. By the same token, middle clouds do not affect the channels around $380 \mathrm{GHz}$. The effect of thin liquid clouds is negligible around $183 \mathrm{GHz}$, whatever their altitude. On average, the $183-\mathrm{GHz}$ band will be contaminated by clouds $\sim 18 \%$ of the total time (corresponding to cirrostratus, altostratus, nimbostratus, and deep convection). The impact of scattering by ice particles increases with frequency, and above $300 \mathrm{GHz}$, channels with weighting functions peaking at or below ice clouds are affected. The $325-\mathrm{GHz}$ band will be affected by clouds for more than $30 \%$ of the time. The 380-GHz band sounding at high altitudes will be less, disrupted by most clouds. The sensitivity to ice clouds at high frequencies can be used to analyze ice clouds.

\section{c. The effect of sensor spatial resolution}

As discussed above, the higher the frequency, the larger the cloud impact for a given gaseous opacity; however, the higher the frequency, the higher the spatial resolution. Considering that cloud amount is scale dependent, what is the change in cloud fraction resulting from a change in spatial resolution? One could imagine that increasing the spatial resolution of an instrument would increase the probability of clear-sky situations, especially in regions dominated by small broken clouds.

Cloud climatologies with different spatial resolution have been compared and the results are similar. The ISCCP cloud climatological data have been compared 

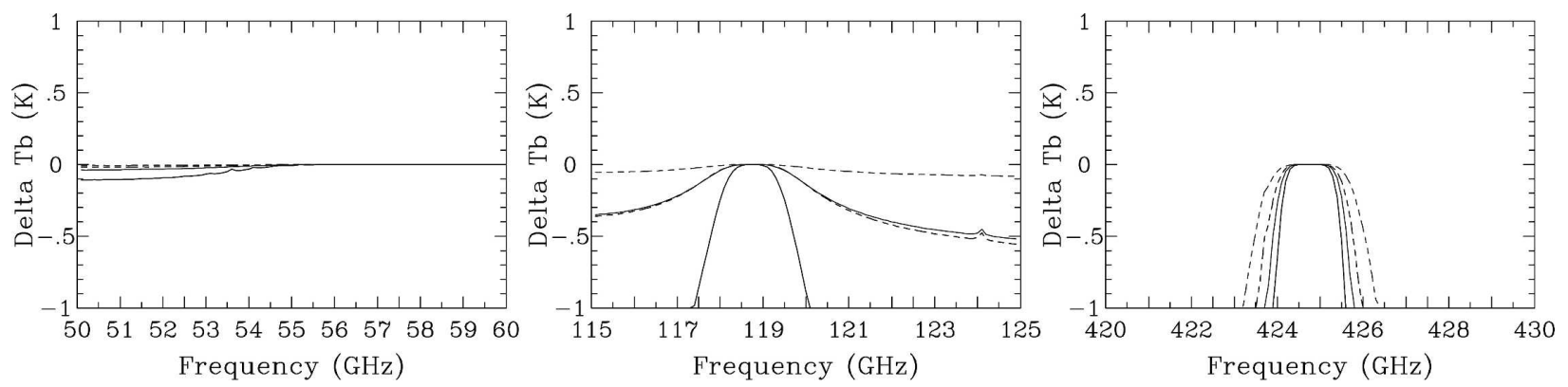

FIG. 6. Sensitivity of $\mathrm{O}_{2}$ lines to ice clouds (DeltaTb $=\mathrm{Tb}$ cloudy sky minus $\mathrm{Tb}$ clear sky), depending on their particle size. Calculations are performed for a standard mean atmosphere over land (emissivity $=1.0$ ), with a high ice cloud between 360 and 300 $\mathrm{hPa}$. Effective radius $=30 \mu \mathrm{m}$ (gray lines) and $60 \mu \mathrm{m}$ (black lines); thin cloud (IWP $=0.037 \mathrm{~kg} \mathrm{~m}^{-2}$, dashed lines) and medium cloud (IWP $=0.241 \mathrm{~kg} \mathrm{~m}^{-2}$, solid lines).

with the surface-based climatological information of Warren et al. (1986) over land and Warren et al. (1988) over ocean. Ground observations correspond to samples of $\sim 100 \mathrm{~m}$ covering an area about $30-50 \mathrm{~km}$ across, which is different from the $30-\mathrm{km}$ sampling of the initial $5-\mathrm{km}$ resolution of the ISCCP observations. The derived cloud amounts are about the same over ocean with just a $\sim 10 \%$ underestimate over land. Usually, clear-sky occurrence is underestimated if the resolution is larger than the most frequent cloud element. In Stubenrauch et al. (2002), ISCCP $30-\mathrm{km}$ pixels are grouped by increasing number and the clear-sky frequency is estimated for each grouping type. The clearsky frequency decreases by less than $5 \%$ for each added pixel, except in the case of stratocumulus, where the decrease is larger. Analysis of all-sky camera images over the eastern United States showed a high correlation between overhead and whole-sky cloud amounts, suggesting that clouds with scales larger than 2-3 km are dominant in those regions (Willand and Steeves 1991). All studies agree that the major difference in cloud amount between climatologies is usually due to the threshold used to detect cloudiness (e.g., Rossow et al. 1985; Rossow and Garder 1993a,b; Nair et al. 1998; Stubenrauch et al. 2002), and not to the spatial resolution of the observations.

Vocabulary tends to emphasize the difference between extended- (stratus) and small- (cumulus) scale
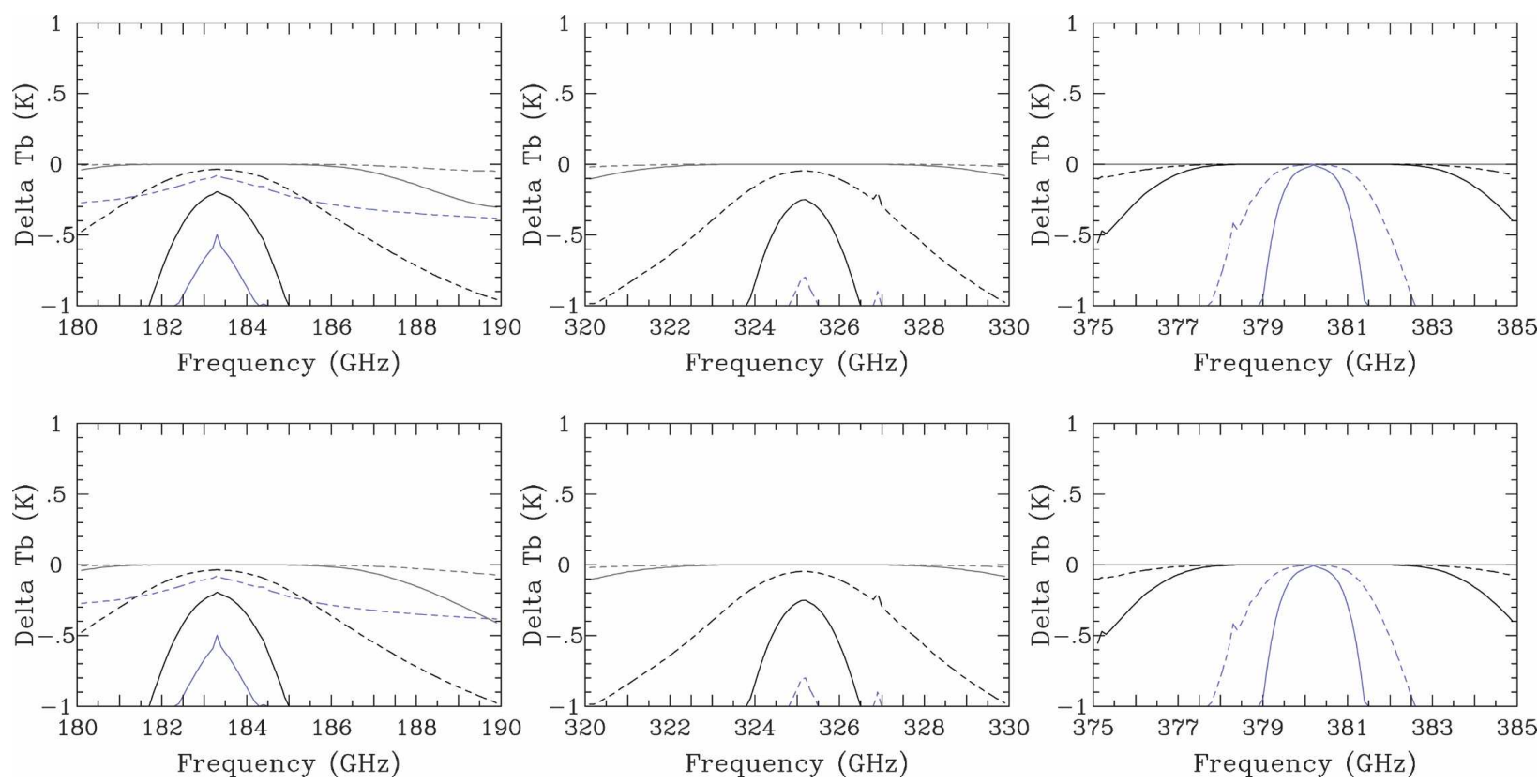

FIG. 7. Same as Fig. 5, but for $\mathrm{H}_{2} \mathrm{O}$. 
clouds, and most studies about resolution effects on cloud amount are focused on fair-weather boundary layer broken clouds that only represent $\sim 20 \%$ of the cloudy situations (i.e., $\sim 13 \%$ of the total clear and cloudy cases). In addition, scattered and broken clouds tend to occur over areas that are much larger in scale than the individual element (Sèze and Rossow 1991a,b); they are often organized at scales larger than $15 \mathrm{~km}$ [shown from three-dimensional cloud modeling (e.g., Ramirez et al 1990), or from satellite observations at high resolution (e.g., Nair et al. 1998)]. The smaller the clouds, the higher the clumping effect. Wielicki and Parker (1992) examined Landsat data, averaged at different resolutions from the nominal $28.5 \mathrm{~m}$ to $8 \mathrm{~km}$, in order to analyze the sensitivity of cloud cover to changing spatial resolution. The resolution effect strongly depends on cloud type and is essentially important for fair-weather boundary layer broken clouds. From 4 to 2 $\mathrm{km}$, changes on the order of $2 \%$ are observed. The change usually increases with decreasing resolution.

Consequently, the probability of clear sky does not increase much with increasing spatial resolution for spatial resolutions of the order of a few kilometers and more. As a consequence, the gain in clear-sky probability resulting from a better spatial resolution at higher radiometric frequencies will not compensate for the increased sensitivity to clouds.

\section{Conclusions}

For temperature soundings in clear sky, the 60- and $118-\mathrm{GHz}$ bands show similar potential with a factorof- 2 better spatial resolution on the ground at $118 \mathrm{GHz}$ for a given antenna size. The $118-\mathrm{GHz}$ band has a slightly larger sensitivity to the water vapor profile than the $60-\mathrm{GHz}$ band does, but this disadvantage could be mitigated by combining temperature and water vapor sounding capabilities. The $424-\mathrm{GHz}$ band could also be used if there is an interest in higher spatial resolution, but only the upper atmosphere above $\sim 500 \mathrm{hPa}$ could be sounded.

Both 60 - and $118-\mathrm{GHz}$ bands will be affected by very thick clouds ( $\sim 7 \%$ of total cases). For the medium liquid clouds ( $\sim 15 \%$ of the total cases), the $118-\mathrm{GHz}$ band will be slightly more affected than the $60-\mathrm{GHz}$ bands, depending on the liquid water content of the cloud. The other clouds (thin liquid clouds and thin and medium ice clouds) will not significantly interfere with the soundings at either 60 or $118 \mathrm{GHz}$. The 424-GHz band is sensitive to ice clouds and can be exploited to detect and characterize the ice phase in clouds. These cloud effects can be detected and accounted for by analyzing the microwave measurements, together with visibleinfrared measurements on the same weather satellites.

The 183- and 325-GHz bands offer similar possibilities for clear-sky humidity soundings with a much better spatial resolution at 325 than at $183 \mathrm{GHz}$ for a given antenna size (a ratio of 325/183), but a reduced sensitivity to the lower atmosphere at $325 \mathrm{GHz}$. Channels around the $380-\mathrm{GHz}$ water vapor line can be added to get information higher in the atmosphere.

The $183-\mathrm{GHz}$ band will be contaminated by clouds for $\sim 18 \%$ of the total time on average. The $325-\mathrm{GHz}$ band will be affected by clouds for more than $30 \%$ of the time. The $384-\mathrm{GHz}$ band sounding at higher altitudes will be less disrupted by most clouds. Information on ice clouds can be provided by channels above 300 GHz.

Acknowledgments. We thank three anonymous reviewers for their very careful reading of the manuscript and for their constructive comments. This study has been partly supported by an ESA contract "Study of future microwave sounders on geostationary and medium earth orbits" in collaboration with EADS Astrium UK. We thank Ulf Klein from ESA for his helpful comments.

\section{REFERENCES}

Chédin, A., D. Pick, R. Rizzi, and P. Schuessel, 1986: Second Generation METEOSAT definition study: Microwave and infra-red vertical sounders. ESA Rep. STR-219, 103 pp.

Davis, C. P., D. L. Wu, C. Emde, J. H. Hiang, R. E. Cofield, and R. S. Harwood, 2005: Cirrus induced polarization in $122 \mathrm{GHz}$ aura Microwave Limb Sounder radiances. Geophys. Res. Lett., 32, L14806, doi:10.1029/2005GL022681.

Evans, K. F., and G. L. Stephens, 1995: Microwave radiative transfer through clouds composed of realistically shaped ice crystals. Part II: Remote sensing of ice clouds. J. Atmos. Sci., 52, 2058-2072.

— A. H. Evans, I. G. Nolt, and B. T. Marshall, 1999: The prospect for remote sensing of cirrus clouds with a submillimeterwave spectrometer. J. Appl. Meteor., 38, 514-525.

_ S. J. Walter, A. J. Heymsfield, and G. M. McFarquhar, 2002: Submillimeter-Wave Cloud Ice Radiometer: Simulations of retrieval algorithm performance. J. Geophys. Res., 107, 4028, doi:10.1029/2001JD000709.

Garand, L., and Coauthors, 2001: Radiance and Jacobian intercomparison of radiative transfer models applied to HIRS and AMSU channels. J. Geophys. Res., 106, 24 017-24 031.

Gasiewski, A. J., 1992: Numerical sensitivity analysis of passive EHF and SMMW channels to tropospheric water vapor, clouds, and precipitation. IEEE Trans. Geosci. Remote Sens., 30, 859-870.

—_, and J. T. Johnson, 1993: Statistical temperature profile retrievals in clear-air using passive $118 \mathrm{GHz} \mathrm{O} 2$ observation. IEEE Trans. Geosci. Remote Sens., 31, 106-115. 
Goutoule, J.-M., C. Prigent, L. Eymard, and U. Klein, 2003: New concept for an ice-cloud sub-millimeter space borne radiometer. Int. Geoscience Remote Sensing Symp., Toulouse, France, IGARSS, 122-125.

Guillou, C., S. J. English, and C. Prigent, 1996: Passive microwave airborne measurements of the sea surface response at 89 and 157 GHz. J. Geophys. Res., 101, 3775-3788.

Han, Q., W. B. Rossow, and A. A. Lacis, 1994: Near-global survey of effective droplet radii in liquid water clouds using ISCCP data. J. Climate, 7, 465-497.

,$- \ldots$, R. Weilch, A. White, and J. Chou, 1995: Validation of satellite retrievals of cloud microphysics and liquid water path using observations from FIRE. J. Atmos. Sci., 52, 41834195.

Klein, M., and A. J. Gasiewski, 2000: Nadir sensitivity of passive millimeter and submillimeter wave channels to clear air temperature and water vapor variations. J. Geophys. Res., 105, 17 481-17 511.

Manabe, T., H. J. Liebe, and G. A. Hufford, 1987: Complex permittivity of water between 0 and 30 THz. Proc. 12th Int. Conf. on Infrared and Millimeter Waves, Orlando, FL, IEEE, 229230.

Miao, J., K.-P. Johnson, S. Buehler, and A. Kokhanovsky, 2003: The potential of polarization measurements from space at $\mathrm{mm}$ and sub-mm wavelengths for determining cirrus cloud parameters. Atmos. Chem. Phys., 3, 39-48.

Mishchenko, M. I., 1991: Light scattering by randomly oriented axially symmetric particles. J. Opt. Soc. Amer., 8, 871-882.

1993: Light scattering by size/shape distributions of randomly oriented axially symmetric particles of a size comparable to a wavelength. Appl. Opt., 32, 4652-4666.

_- J. Hovenier, and L. Travis, 2000: Light Scattering by NonSpherical Particles. Elsevier, 690 pp.

Nair, U. S., R. C. Weger, K. S. Kuo, and R. M. Welch, 1998: Clustering, randomness, and regularity in cloud fields. 5. The nature of regular cumulus cloud fields. J. Geophys. Res., 103, 11 363-11 380.

Pardo, J. R., J. Cernicharo, and E. Serabyn, 2001a: Atmospheric Transmission at Microwaves (ATM): An improved model for $\mathrm{mm} / \mathrm{submm}$ applications. IEEE Trans. Antennas Propag., 49, 1683-1694.

_ _ E. Serabyn, and J. Cernicharo, 2001b: Submillimeter atmospheric transmission measurements on Mauna Kea during extremely dry El Niño conditions: Implications for broadband opacity contributions. J. Quant. Spectrosc. Radiat. Transfer, 68, 419-433.

- M. Ridal, D. P. Murtagh, and J. Cernicharo, 2002: Microwave temperature and pressure measurements with the Odin satellite: I. Observational method. Can. J. Phys., 80, 443-454.

—, M. Wiedner, E. Serabyn, C. D. Wilson, C. Cunningham, R. E. Hills, and J. Cernicharo, 2004: Side-by-side comparison of Fourier transform spectroscopy and water vapor radiometry as tools for the calibration of $\mathrm{mm} / \mathrm{submm}$ ground-based observatories. Astrophys. J. Suppl., 153, 363-367.

— E. Serabyn, M. C. Wiedner, and J. Cernicharo, 2005: Measured telluric continuum-like opacity beyond $1 \mathrm{THz} . J$. Quant. Spectrosc. Radiat. Transfer, 96, 537-545.

Prigent, C., W. B. Rossow, and E. Matthews, 1997: Microwave land surface emissivities estimated from SSM/I observation. J. Geophys. Res., 102, 21 867-21 890.
- J. R. Pardo, M. I. Mishchenko, and W. B. Rossow, 2001: Microwave polarized signatures generated within cloud systems: SSM/I observations interpreted with radiative transfer simulations. J. Geophys. Res., 106, 28 243-28 258.

- E. Defer, J. R. Pardo, C. Pearl, W. B. Rossow, and J.-P. Pinty, 2005a: Relations of polarized scattering signatures observed by the TRMM Microwave Instrument with electrical processes in cloud systems. Geophys. Res. Lett., 32, L04810, doi:10.1029/2004GL022225.

—, F. Chevallier, F. Karbou, P. Bauer, and G. Kelly, 2005b: AMSU-A land surface emissivity estimation for numerical weather prediction assimilation schemes. J. Appl. Meteor., 44, 416-426.

Ramirez, J. A., R. L. Bras, and K. A. Emanuel, 1990: Stabilization functions of unforced cumulus clouds: Their nature and components. J. Geophys. Res., 95, 2047-2059.

Ridal, M., J. R. Pardo, D. P. Murtagh, F. Merino, and L. Pagani, 2002: Microwave temperature and pressure measurements with the Odin satellite: II. Retrieval method. Can. J. Phys., 80, 454-467.

Rossow, W. B., and R. A. Schiffer, 1991: ISCCP cloud data product. Bull. Amer. Meteor. Soc., 72, 2-20.

_ measurements of infrared and visible radiances for ISCCP. J. Climate, 6, 2341-2369.

— , and — 1993b: Validation of ISCCP cloud detection. J. Climate, 6, 2370-2393.

_ from ISCCP. Bull. Amer. Meteor. Soc., 80, 2261-2287.

_ matology Project (ISCCP) Web site: An online resource for research. Bull. Amer. Meteor. Soc., 85, 167-172.

- , and Coauthors, 1985: ISCCP cloud algorithm intercomparison. J. Climate Appl. Meteor., 24, 877-903.

__, A. W. Walker, and L. C. Garder, 1996: Comparison of ISCCP and other cloud amount. J. Climate, 6, 2394-2418.

Sèze, G., and W. B. Rossow, 1991a: Time-cumulated visible and infrared radiance histograms used as descriptors of surface and cloud variations. Int. J. Remote Sens., 12, 877-920.

—, and _ 1991b: Effects of satellite data resolution on measuring the space/time variations of surface and clouds. Int. $J$. Remote Sens., 12, 921-952.

Staelin, D. H., A. J. Gasiewski, J. P. Kerekes, M. W. Shields, and F. J. Solman III, 1998: Concept proposal for a Geostationary Microwave (GEM) Observatory. MIT, Prepared for the NASA/NOAA Advanced Geostationary Sensor Program, 23 pp.

Stubenrauch, C. J., R. Holz, A. Chédin, D. L. Mitchell, and A. J. Baran, 1999: Retrieval of cirrus ice crystal sizes from 8.3 and 11.1 microns emissivities determined by the improved initialization inversion of TIROS-N Operational Vertical Sounder. J. Geophys. Res., 104, 31 793-31 808.

—, V. Briand, and W. B. Rossow, 2002: The role of clear-sky identification in the study of cloud radiative effects: Combined analysis from ISCCP and the Scanner of Radiation Budget. J. Appl. Meteor., 41, 396-412.

Warren, S. G., 1984: Optical constants of ice from the ultraviolet to the microwave. Appl. Opt., 23, 1206-1225.

— C. C. Hahn, J. London, R. M. Chervin, and R. L. Jenne, 1986: Global distribution of total cloud and cloud type amounts over land. NCAR Tech. Note TN-273, STR/DOE Tech. Rep. ER/60085-HI, 29 pp. 
,,$-- \quad$ and -1988 : Global distribution of total cloud and cloud type amounts over the ocean. NCAR Tech. Note TN-317, STR/DOE Tech. Rep. ER-0406, 41 pp.

Waters, J., and Coauthors, 1999: The UARS and EOS Microwave Limb Sounder (MLS) experiments. J. Atmos. Sci., 56, 194-218.

Wiedner, M., C. Prigent, J. R. Pardo, O. Nuissier, J.-P. Chaboureau, J.-P. Pinty, and P. Mascart, 2004: Modeling of passive microwave responses in convective situations using out- puts from mesoscale models: Comparison with TRMM/TMI satellite observations. J. Geophys. Res., 109, D06214, doi:10.1029/2003/JD004280.

Wielicki, B. A., and L. Parker, 1992: On the determination of cloud cover from satellite sensors: The effect of sensor spatial resolution. J. Geophys. Res., 97, 12 799-12 823.

Willand, J. H., and J. Steeves, 1991: Sky-cover correlation within a sky dome. J. Appl. Meteor., 30, 1037-1039. 